\title{
EVALUATION OF IN VITRO INHIBITION OF PROTON-PUMP BY NANOSILVER PARTICLES SYNTHESIZED USING SEEDS OF ANETHUM GRAVEOLENS
}

\author{
MENAGA S, KRIPA KG, SANGEETHA R* \\ Department of Biochemistry, School of Life Sciences, Vels University, Chennai, Tamil Nadu, India. Email: sara_dna@yahoo.co.in
}

Received: 2 January 2017, Revised and Accepted: 14 January 2017

ABSTRACT

Objective: The green synthesis of nanoparticles is gaining popularity owing to its several advantages over the chemical methods. This study reports the biosynthesis of nanosilver mediated by the extract of the seeds of Anethum graveolens.

Methods: Green synthesis of silver nanoparticles (AgNPs) was carried out using the aqueous extract of the seeds of $A$. graveolens (AESE). Microwave irradiation was done to hasten the process. The AgNPs synthesized were physically characterized using ultraviolet-visible spectroscopy, X-ray diffraction, Fourier transform infrared spectroscopy and scanning electron microscopy. The potential of the nanosilver particles in inhibiting $\mathrm{H}^{+} \mathrm{K}^{+}$ adenosine triphosphatase (proton pump) was evaluated.

Results: The nanosilver particles were initially subjected to characterization studies to ascertain their shape, size and surface composition. The particles were found to be crystalline, spherical of size $30-35 \mathrm{~nm}$. The nanosilver particles inhibited the activity of proton pump by $67 \%$ and thus were as potential as the standard drug, omeprazole.

Conclusion: The seeds have several pharmacological benefits including its gastroprotective potential and hence the nanosilver particles synthesized using AESE was assessed for its gastroprotective potential. The results of this study prove that the nanosilver synthesized by green technology can inhibit proton-pump and thus act as promising gastroprotective agents.

Keywords: Green synthesis, Nanoparticles, Anethum graveolens, Proton pump inhibition.

(c) 2017 The Authors. Published by Innovare Academic Sciences Pvt Ltd. This is an open access article under the CC BY license (http://creativecommons. org/licenses/by/4. 0/) DOI: http://dx.doi.org/10.22159/ajpcr.2017.v10i6.16896

\section{INTRODUCTION}

Nanotechnology is now considered to be a growing area of research in life sciences especially in health and biomedical industry [1]. Nanotechnology is concerned with the synthesis of nanoparticles of variable size, shapes and composition and their potential use for benefits for humans [2]

Nanosilver has many medical applications including diagnosis, treatment, and medical devices. Nanosilver is used for coating medical tools and materials, in wound dressings, diabetic socks, used in the areas of surgery, in orthopedics, dentistry and eye care [3].

Traditionally, nanoparticles are synthesized by chemical means. However, greener approaches help to overcome the deleterious side effects of traditional methods. Eco-friendly methods using plants and microbes for the biosynthesis of nanoparticles have several advantages. Green synthesis of nanoparticles is cost-effective, environment-friendly and offers control over crystal growth and size. The active constituents of medicinal plants cap the nanoparticles during green synthesis process, and thus this process fortifies the clinical use of nanoparticles [4].

Anethum graveolens also known as Peucedanum graveolens belongs to the family Umbelliferae. It is also known as Sthatapushpi in Sanskrit and as Dill in English. A. graveolens L. (dill) is a sparse looking plant with feathery leaves and tiny yellow flowers. Many pharmacological effects such as antimicrobial, antihyperlipidemic, and antihypercholesterolemic activities of dill extracts have been reported. As a folk remedy, dill is considered for some gastrointestinal ailments such as flatulence, indigestion, stomach ache, and colic. Dill fruit has an antispasmodic effect on the smooth muscles of the gastrointestinal tract [5]. The objective of this study was the biosynthesis of nanosilver using the extract of $A$. graveolens and investigation of in vitro proton pump inhibition by the nanoparticles. This study is of first of its kind as the inhibition of proton pump by nanoparticles has not been reported so far.

\section{METHODS}

\section{Plant materials}

A. graveolens seeds were collected from a Local Herbal Practitioner. The seeds were identified by Dr. J. Jayaraman, Plant Anatomy Research Center, Tambaram, Chennai, India (Voucher No. PARC/2015/1565). The seeds were air dried under shade, powdered mechanically and stored in airtight containers.

\section{Preparation of extracts}

Coarsely powdered material of $A$. graveolens seeds was subjected to aqueous extraction. $25 \mathrm{~g}$ of coarse powder was mixed in $200 \mathrm{ml}$ water and kept on a magnetic stirrer for $24 \mathrm{hrs}$ after which it was filtered and the filtrate was evaporated to dryness and stored in airtight container.

\section{Synthesis of nanoparticles}

Aqueous solution (1 mM) of silver nitrate (Hi Media) was used for the green synthesis of silver nanoparticles (AgNPs). $100 \mathrm{ml}$ of silver nitrate solution was taken in an Erlenmeyer flask to which $20 \mathrm{ml}$ of plant extract $(\mathrm{mg} / \mathrm{ml})$ was added. The flask was kept in a microwave oven at $300 \mathrm{~W}$ for 5 minutes (discontinuously) and the change in the color of the solution from faint brown to colloidal brown was monitored. Samples were removed periodically at 15 minutes interval for $1 \mathrm{hr}$ and ultraviolet-visible (UV-Vis) spectra were obtained. The solution was kept in the dark for $24 \mathrm{hrs}$ for complete reduction of silver. The solution of AgNPs was freeze-dried and stored for further studies.

\section{UV-Vis spectra analysis}

The samples removed periodically were studied for the reduction of silver by scanning between 300 and $800 \mathrm{~nm}$ in a spectrophotometer (Cary 5E model with $1 \mathrm{~nm}$ resolution). 
Characterization of nanoparticles

\section{Fourier transform infrared (FTIR) spectroscopy}

FTIR analysis of the freeze-dried nanoparticles was carried out by the potassium bromide method. The spectrum was recorded using PerkinElmer Spectrum-One instrument, and 256 scans were made in the range of $400-4000 / \mathrm{cm}$ with a resolution of $4 / \mathrm{cm}$.

\section{Scanning electron microscopy (SEM) analysis}

SEM analysis was performed using Quanta 200 FEG scanning electron microscope. Thin films of the sample were prepared on a carbon-coated copper grid, and then the film on the SEM grid was allowed to dry by putting it under a mercury lamp for 5 minutes.

\section{$\mathrm{H}^{+} \mathrm{K}^{+}$adenosine triphosphatase (ATPase) inhibition}

Gastric microsomes were isolated from goat stomach. The stomach was removed, cut open and washed with ice-cold buffered saline. The mucosa was scrapped and homogenized in $20 \mathrm{mM}$ Tris $\mathrm{HCl}$ (pH7.4), in a Potter-Elvehjem homogenizer at $200 \mathrm{rpm}$. Crude microsomes were harvested as the pellet sedimented at 10 minutes and 13,000 g. $\mathrm{H}^{+} \mathrm{K}^{+}$ ATPase enriched microsomes were collected and stored at $-20^{\circ} \mathrm{C}$ until use.

The reaction mixture consisting of $0.4 \mathrm{ml}$ extract and $0.2 \mathrm{ml}$ enzyme source was pre-incubated for 30 minutes at $37^{\circ} \mathrm{C}$. The reaction was initiated by adding $0.2 \mathrm{ml}$ Tris $\mathrm{HCl}(\mathrm{pH} 7.4), 0.2 \mathrm{ml} \mathrm{MgCl}_{2}(2 \mathrm{mM}), 0.2 \mathrm{ml}$ $\mathrm{KCl}(10 \mathrm{mM})$, and $0.2 \mathrm{ml}$ ATP $(2 \mathrm{mM})$. After incubation for 30 minutes at $37^{\circ} \mathrm{C}$, the reaction was terminated by the addition of $1 \mathrm{ml}$ of tricarboxylic acid $(10 \%)$. The reaction mixture was centrifuged at $6000 \mathrm{~g}$ for 10 minutes, and the supernatant was analyzed for the presence of inorganic phosphate by Fiske Subbarow method [6]. Enzyme activity was calculated as the micromoles of inorganic phosphate released per hour. The efficacy of the extract was compared with the standard proton pump inhibitor, omeprazole.

\section{RESULTS}

Nanoparticles are found to have diverse applications and the medical applications of nanoparticles are highly promising. The nanoparticles synthesized using green technology are found to be effective than those produced by chemical methods [7]. In this research work, the synthesis of AgNPs using the extract of the seeds of A. graveolens was studied. To fasten the process, heating using microwaves was adopted. An even heat transfer is critical for the formation of homogenous AgNPs. This is provided by microwave heating.

Immediately after the addition of $A$. graveolens seeds extract to the silver nitrate solution, a light yellowish color was observed which changed to dark brown color on subjecting to microwave irradiation. This change of color indicates that the reduction of silver particles has taken place. Silver nitrate solution without the addition of plant extract was exposed to microwaves for the same duration and the change in color was observed. There was no change in color, and this showed that the reduction of silver was by the plant extract and not by the microwaves. The microwave-assisted method is much faster than the earlier conventional studies with other biological routes. The time required for the conventional synthesis of AgNPs from other plants was 2-4 hrs and from bacteria was 24-120 hrs and thus are rather slow. At room temperature, the stability of the synthesized AgNPs was observed for 12 weeks during the period of the study, and this is much greater than the particles produced from other biological methods [8].

\section{UV-vis spectroscopy}

UV-vis spectroscopy is one of the most widely used techniques for structural characterization of AgNPs. The spectrum depends on the particle size and the constituents adsorbed on the surface of the nanoparticles. Only a single surface plasmon resonance (SPR) band is expected in the spectra of spherical nanoparticles, whereas nonspherical particles have two or more SPR bands depending on the shape of the particles. The number of peaks varies with the symmetry of the nanoparticles. Thus, spherical nanoparticles, disks, and triangular nanoplates of silver show one, two, and more peaks, respectively [9].

In this study, a single peak at 430-455 nm was observed with the AgNPs synthesized (Fig. 1). The absence of a peak between 400 and $500 \mathrm{~nm}$ in the spectrum of the colloidal silver nitrate solution which was devoid of plant extract indicated the absence of nanoparticles and thus ensured the effect of plant extract in the biogenic synthesis of nanoparticles.

\section{X-ray diffraction (XRD)}

XRD study was done to confirm the crystalline nature of the particles and the XRD pattern obtained is shown in Fig. 2. The exact nature of the silver particles formed can be deduced from the XRD spectrum of the sample. XRD spectra of pure crystalline silver structures have been published by the Joint Committee on Powder Diffraction Standards (file no. 04-0783).

A comparison of our XRD spectrum with the standard confirms that the silver particles produced by green synthesis method were in the form of nanocrystals, as indicated by the peaks at 2 values of such peaks $38.43^{\circ}$, $44.40^{\circ}$ and $67.57^{\circ}$, corresponding to 111,200 and 220 planes for silver, respectively (Fig. 2)

The XRD pattern thus clearly shows that the AgNPs are crystalline in nature. In addition to the Bragg peak representative of FCC silver nanocrystals, no additional and unassigned peaks were observed suggesting the absence of crystallization of bio-organic phase occurs on the surface of the AgNPs [10].

\section{FTIR}

FTIR measurements were carried out to identify the possible biomolecules accountable for the reduction of the $\mathrm{Ag}^{+}$ions, stabilization of nanoparticles and the capping of AgNPs by the active constituents present in the leaf extract.

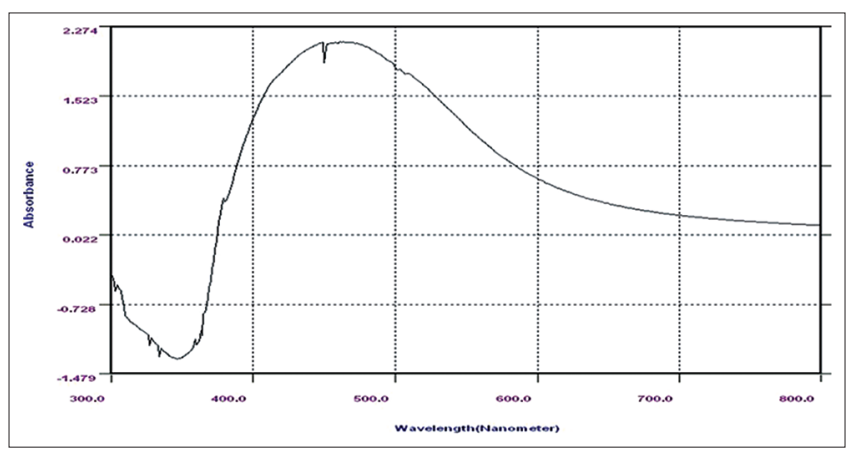

Fig. 1: Ultraviolet-visible spectrum of aqueous silver nitrate with aqueous extract of the seeds of Anethum graveolens

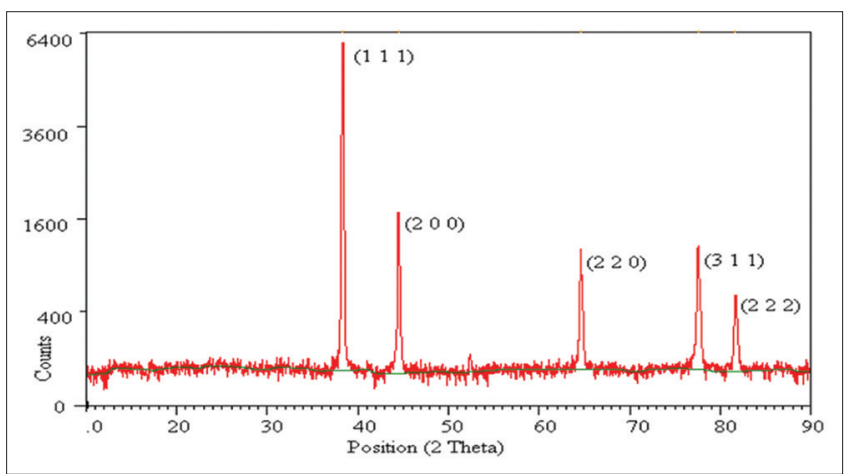

Fig. 2: X-ray diffraction pattern of silver nanoparticles synthesized using aqueous extract of the seeds of Anethum graveolens 
Appearance of broad peak at wave number 3400 in the nanoparticle and plant extract spectrum indicates the presence of phenolic hydroxyl group $(\mathrm{OH})$ belonging to flavonoids and tannins. The peak of $870 / \mathrm{cm}$ represents the aromatic ring $\mathrm{C}-\mathrm{H}$ and indicates the presence of free catechin. This also proves the attachment of some polyphenolic components on to AgNPs. Thus, the polyphenols attached to AgNPs may have at least one aromatic ring. The existence of peaks at $1010 / \mathrm{cm}$ may be attributed to the presence of aliphatic amines and those at $1100-1200 / \mathrm{cm}$ indicate groups of polyphenols such as catechin gallate, epicatechin gallate, and theaflavin [11]. The presence of constituents such as proteins on the surface of nanoparticles could therefore prevent agglomeration of the metal nanoparticles (Fig. 3).

\section{SEM}

SEM image showing the high-density AgNPs synthesized in the presence of aqueous extract of the seeds of A. graveolens (AESE) further confirmed the formation of silver nanostructures. The analysis showed particles with size between 30 and $35 \mathrm{~nm}$ which were spherical in shape (Fig. 4)

\section{Proton pump inhibition by AgNPs synthesized using AESE}

Ulcer is a common lifestyle associated problem attributed to several factors such as stress, exposure to bacterial infection and the use of non-steroidal anti-inflammatory drugs. Mucosal damage is the initial step in ulcer development and is correlated with oxidative stress by the generation of reactive oxygen species (ROS) and hypersecretion of $\mathrm{HCl}$ through $\mathrm{H}^{+} \mathrm{K}^{+}$ATPase action. The best approach to control ulceration may be by scavenging ROS in the stomach and inhibiting $\mathrm{H}^{+} \mathrm{K}^{+}$ATPase, a proton-pump for acid secretion in the parietal cells of gastric mucosa; The antisecretory drugs such as $\mathrm{H}^{+} \mathrm{K}^{+}$ATPase inhibitors - omeprazole, lansoprazole, ranitidine, and histamine $\mathrm{H}_{2}$ receptor blockers being used to control acid secretion produce adverse side effects on human health. Proton-pump inhibitors have revolutionized the treatment of peptic ulcer; however, a complete cure for this disease has not been envisioned. The long-term use of these drugs leads to various adverse side effects [12]. Thus, there is an urgent requirement to identify more effective and safe anti-ulcer agents. A widespread search has been initiated and researchers are exploring safer anti-ulcer therapies. Herbs, medicinal plants and crude drug substances are considered to be a potential source to combat various diseases including gastric ulcer. A. graveolens possesses various medicinal values and the folklore claims of those medicinal values have been proved by various researchers. The extracts of seeds of A. graveolens have been proved to protect the gastric mucosal lining in ethanol-induced gastric lesions in rats [13], and the objective of our study was to analyze the inhibitory effect of the AESE on gastric $\mathrm{H}^{+} \mathrm{K}^{+}$ATPase and thus establish its gastroprotective effect indirectly.

The percentage of inhibition of proton-pump of the extract was $67 \%$, and the effect was comparable with the standard proton-pump inhibitor drug, omeprazole which exhibited $71 \%$ inhibition (Fig. 5). The study thus opens a new avenue for an exhaustive investigation on the in vivo use of A. graveolens as an anti-ulcer agent.

There are reports on the gastroprotective effect of nanoparticles synthesized by green technology; however, the gastroprotective effect was mediated through the inhibition of Helicobacter pylori [14] and modulation of apoptotic pathway [15]. The proton pump inhibition by nanoparticles has not been established, and thus this study of proton pump inhibition by the nanosilver biosynthesized using a plant extract is novel and the in vivo gastroprotective effect of these nanosilver particles needs to be validated.

\section{CONCLUSION}

The AESE was used to synthesize AgNPs and biogenesis process was assisted by microwaves. The heat generated by the microwaves proved to reduce the production time. Characterization of AgNPs was done by analyzing the UV-Vis, XRD and FTIR spectra. These studies confirmed the formation of AgNPs and also proved the presence of plant

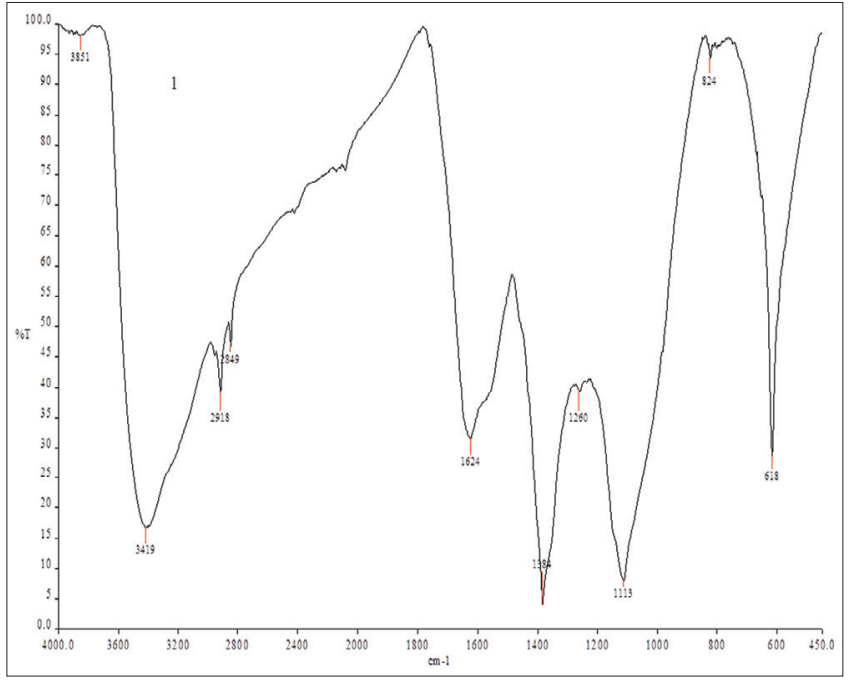

Fig. 3: Fourier transform infrared spectrum of silver nanoparticles synthesized using aqueous extract of the seeds of Anethum graveolens

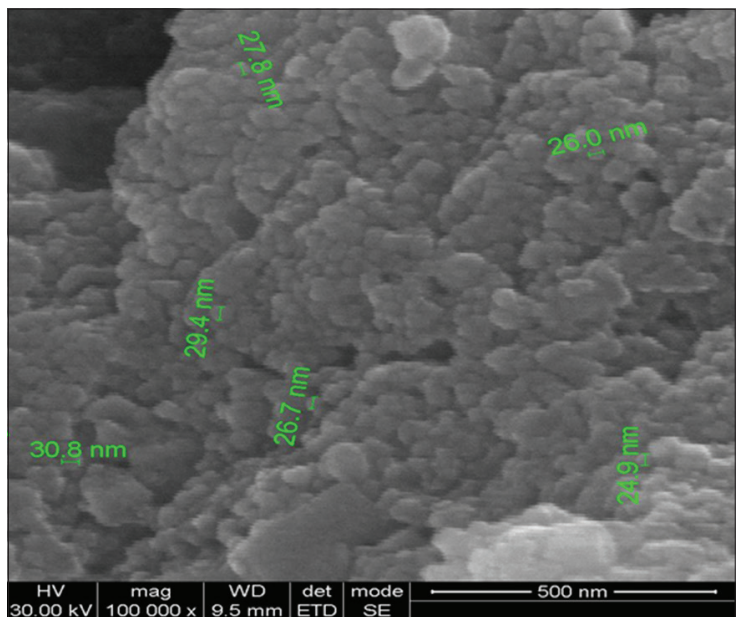

Fig. 4: Scanning electron microscopy micrograph of silver nanoparticles synthesized using aqueous extract of the seeds of Anethum graveolens

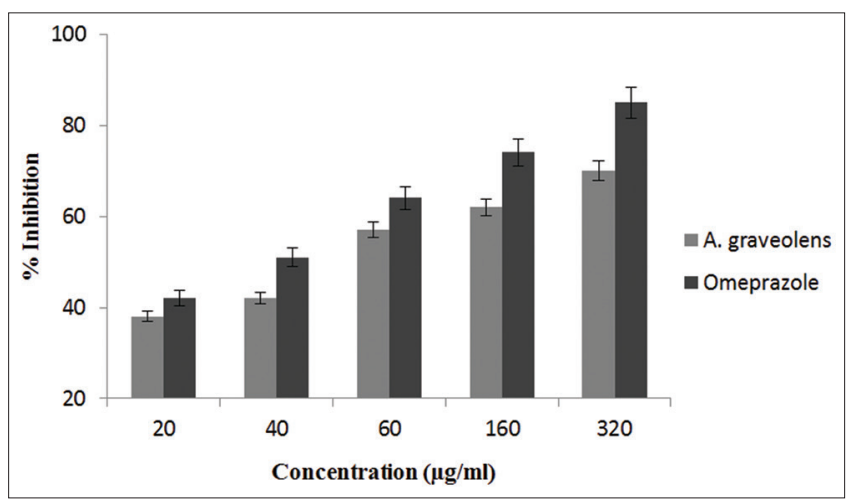

Fig. 5: In vitro inhibition of $\mathrm{H}^{+} \mathrm{K}^{+}$adenosine triphosphatase activity by aqueous extract of the seeds of Anethum graveolens and omeprazole. Values expressed are mean $\pm S D$ of three independent experiments. AESE: Aqueous extract of the seeds of Anethum graveolens. SD: Standard Deviation

secondary metabolites on the surface of the nanoparticles. The size of the nanoparticles was found to be $30-35 \mathrm{~nm}$ as suggested by SEM study. 
A. graveolens seeds have been claimed to possess gastroprotective activity, and this study confirmed that the AgNPs synthesized using the extract of $A$. graveolens seeds can inhibit the proton pump of the gastric mucosal cells and thereby protect the gastric lining.

\section{REFERENCES}

1. Rao ML, Bhumi G, Savithramma N. Green synthesis of silver nanoparticles by Allamanda Cathartica L. leaf extract and evaluation for antimicrobial activity. Int J Pharm Sci Nanotechnol 2013;6:2260-8.

2. Dubey MS, Kushwah BS. Green synthesis of nanosilver particles from extract of Eucalyptus hybrida (safeda) leaf. Dig J Nanomater Biostruct 2009;4:537-43.

3. Tolaymat TM, El Badawy AM, Genaidy A, Scheckel KG, Luxton TP Suidan M. An evidence-based environmental perspective of manufactured silver nanoparticle in syntheses and applications: A systematic review and critical appraisal of peer-reviewed scientific papers. Sci Total Environ 2010;408:999-1006.

4. Sangeetha R, Niranjan P, Dhanalakshmi N. Characterization of silver nanoparticles synthesized using the extract of the leaves of Tridax procumbens. Res J Med Plant 2016;10:159-66.

5. Jana A, Shekhawat GS. Anethum graveolens: An Indian traditional medicinal herb and spice. Pharmacogn Rev 2010;4:179-84

6. Fiske $\mathrm{CH}$, Subbarow Y. The colorimetric determination of phosphorous. J Biol Chem 1925;66:375-400.

7. Srikar SK, Giri DD, Pal DB, Mishra PK, Upadhyay SN. Green synthesis of silver nanoparticles: A review. Green Sustain Chem 2016;6:34-56.

8. Sadeghi B, Gholamhoseinpoor F. A study on the stability and green synthesis of silver nanoparticles using Ziziphora tenuior $(\mathrm{Zt})$ extract at room temperature. Spectrochim Acta A Mol Biomol Spectrosc 2015;134:310-5.

9. Kalainila P, Subha V, Ravindran RS, Renganathan S. Synthesis and characterization of silver nanoparticle from Erythrina indica. Asian J Pharm Clin Res 2014;7:39-43.

10. Sosa IO, Noguez C, Barrera RG. Optical properties of metal nanoparticles with arbitrary shapes. J Phys Chem 2003;13:62-9.

11. Ahmed A, Saifullah F, Ahmad M, Swami BL, Ikram S. Green synthesis of silver nanoparticles using Azadirachta indica aqueous leaf extract. J Rad Res Appl Sci 2016;9:1-7.

12. Chellappan DR, Pemiah B. Pharmacognostical, phytochemical and in vivo gastroprotective investigation of Gmelina arborea. Int J Pharm Pharm Sci 2014;6(4):153-7.

13. Ali EA. The pharmacological importance of Anethum graveolens: A review. Int J Pharm Pharm Sci 2014;6(4):11-3.

14. Sironmani TA. Therapeutic potential of neem synthesized silver nanoparticles on human gastric cancer cells in vitro. World J Nano Sci Eng 2016;6:90-110.

15. Amin M, Anwar F, Janjua MR, Iqbal MA, Rashid U. Green synthesis of silver nanoparticles through reduction with Solanum xanthocarpum L. berry extract: Characterization, antimicrobial and urease inhibitory activities against Helicobacter pylori. Int J Mol Sci 2012;13(8):9923-41. 